\title{
CME: Achtung, Lamelle! Pilzvergiftungen in der Praxis
}

\section{CME: Mushroom Poisoning in the Family Practice}

\author{
Katharina Schenk-Jäger ${ }^{1,2}$, Mirjam Gessler ${ }^{1}$ und Stefan Weiler ${ }^{1}$ \\ ${ }^{1} T o x$ Info Suisse, Nationale Beratungsstelle für Vergiftungen, Assoziiertes Institut der Universität Zürich, Zürich \\ ${ }^{2}$ Verband Schweizerischer Vereine für Pilzkunde VSVP, Niederurnen
}

\begin{abstract}
Zusammenfassung: In der ärztlichen Praxis die banalen Unverträglichkeiten nach Konsum von Speisepilzen von einer sich anbahnenden Vergiftung mit potenziell tödlichem Ausgang zu unterscheiden ist nicht trivial. Trotzdem gibt es einige Kriterien, an denen man sich orientieren kann: Für eine Vergiftung mit Amatoxinen, den Giftstoffen z.B. im Grünen Knollenblätterpilz (Amanita phalloides) spricht eine Latenz von sechs Stunden oder mehr zwischen dem Konsum von nicht durch Fachpersonen kontrollierten Lamellenpilzen und dem Beginn meist heftiger Brechdurchfälle. Auch wenn die Therapieoptionen kontrovers diskutiert werden, hat sich die zeitnahe antidotale Behandlung mit Silibinin als zielführend erwiesen.
\end{abstract}

Schlüsselwörter: Pilzvergiftung, Lamellenpilz, Silibinin

\begin{abstract}
In the general medical practice, it is not trivial to distinguish between banal intolerances after consumption of edible mushrooms and the initial symptoms of poisoning with potentially fatal outcome. Nevertheless, there are some criteria that can be used as clinical guidance: A latency of six hours or more between the consumption of gilled mushrooms that have not been checked by experts and the onset of mostly severe vomiting and diarrhea is indicative of poisoning with amatoxins, the toxins i.e. in death caps (Amanita phalloides). Although the therapeutic options are controversial, prompt antidotal treatment with silibinin has proven to be effective.
\end{abstract}

Keywords: Mushroom poisoning, lamellar fungus, silibinin

Das Pilzesammeln erfreut sich ungebrochener Beliebtheit, die selbst gesammelten Pilze schmecken nach getaner Arbeit besonders gut. Beschwerden treten dann auf, wenn erstens an sich essbare Pilze nicht korrekt zubereitet oder gelagert wurden oder zweitens giftige Pilze aus nicht fachlich kontrolliertem Sammelgut gegessen werden. Im ersten Fall handelt es sich um ein häufiges Ereignis, das nicht eine Vergiftung im engeren Sinn ist. Die Symptome können durch Schwerverdaulichkeit, ungenügend lange Kochdauer oder verdorbene Pilze bedingt sein $[1,2]$. Im zweiten Fall handelt es sich um eine Vergiftung im engeren Sinn, da die Symptome durch Toxine ausgelöst werden, die in einigen Fällen bekannt sind, wie zum Beispiel die Amatoxine aus dem Grünen Knollenblätterpilz. Es gibt aber auch Vergiftungssyndrome mit einem sehr spezifischen Vergiftungsbild, bei denen das Toxin strukturell nicht bekannt ist. Ein Beispiel dafür ist das Morchellasyndrom mit seinen zerebellären Symptomen [3].

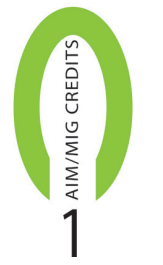

Die Schweizerische Gesellschaft für Allgemeine Innere Medizin (SGAIM/SGIM) anerkennt die CME der «Praxis» als Kernfortbildung AIM und vergibt pro CME AIM 1 Credit.
Initiale Vergiftungssymptome nach Pilzgenuss umfassen am häufigsten Magen-Darm-Symptome, seltener Symptome des zentralen Nervensystems. Ob im Fall von gastrointestinalen Beschwerden mit weiteren Organschäden - insbesondere Leberschäden - gerechnet werden muss, kann anhand der Latenzzeit zwischen Mahlzeit und Beginn der Symptome und Angaben zu den verzehrten Pilzen abgeschätzt werden. Treten die Symptome innerhalb von vier Stunden nach der Mahlzeit auf, handelt es sich meist um eine «Unverträglichkeit» von Speisepilzen oder um ein gastrointestinales Frühsyndrom, das durch ungeniessbare oder magendarmgiftige Pilze verursacht wird. Ist die Latenzzeit jedoch länger, besteht der Verdacht auf eine Amatoxinvergiftung, insbesondere nach Konsum von Lamellenpilzen. Amatoxine sind im Grünen Knollenblätterpilz (Amanita phalloides; Abb. 1), im Kegelhütigen Knollenblätterpilz (Amanita virosa) sowie im Frühjahrs-Knollenblätterpilz (Amanita verna), aber auch in gewissen Arten von Giftschirmlingen (Lepiota sp.) oder 


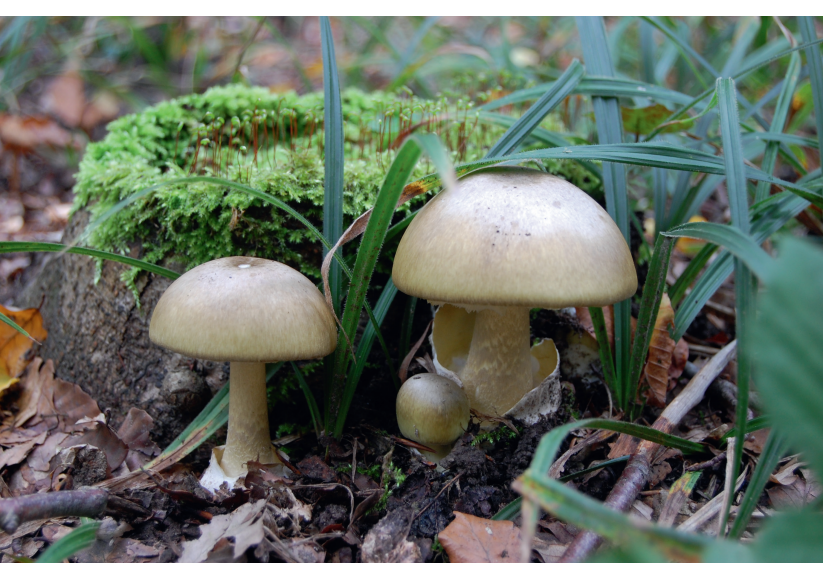

Abbildung 1. Amanita phalloides (Grüner Knollenblätterpilz). Foto: Katharina Schenk-Jäger

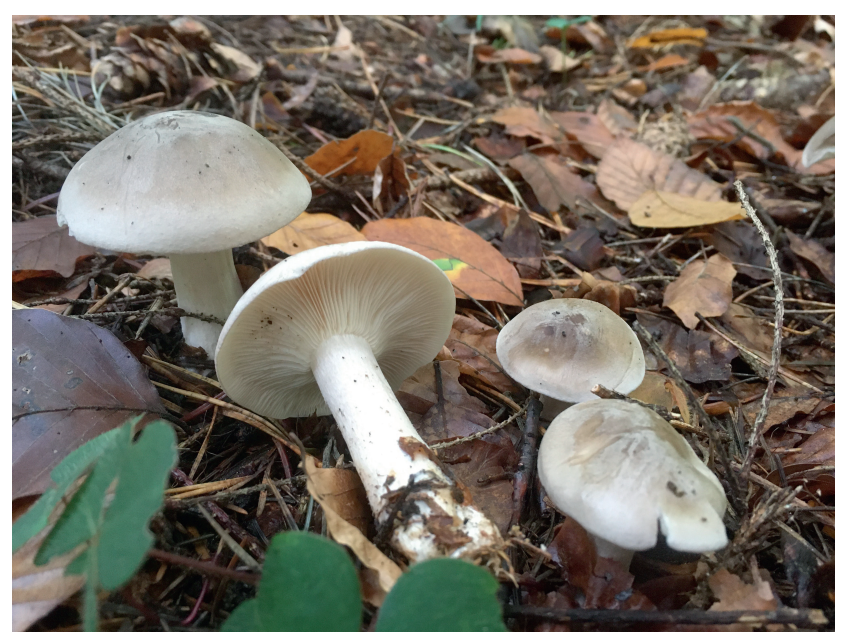

Abbildung 3. Lamellen: Clitocybe nebularis (Nebelkappe).

Foto: Katharina Schenk-Jäger

Gifthäublingen (Galerina sp.) enthalten. Dass im Fall eines Mischpilzgerichts, bestehend aus ungeniessbaren und amatoxinhaltigen Pilzen die durch die ungeniessbaren Pilze verursachte kurze Latenzzeit eine Amatoxinvergiftung maskieren kann, ist denkbar, im praktischen Alltag aber eher eine Seltenheit.

Ein analytischer Nachweis für Amatoxine ist zumindest während der Pilzsaison, also meist von August bis Oktober, in Zentrumsspitälern in der Regel verfügbar. Wegen der potenziellen Lebensgefahr muss jedoch oft mit der antidotalen Therapie begonnen werden, ohne die Resultate der Analytik abzuwarten. Zur Risikobeurteilung hilfreich kann zudem die Einbeziehung von Tox Info Suisse sowie einer Notfall-Pilzexpertin/eines NotfallPilzexperten sein.

Die verschiedenen Fruchtschichten von Pilzen sind in den Abbildungen 2-6 illustriert.

\section{Klinik und Komplikationen}

Pilzvergiftungen lassen sich anhand verschiedener Parameter in Gruppen einteilen. Am gängigsten ist die Ein-

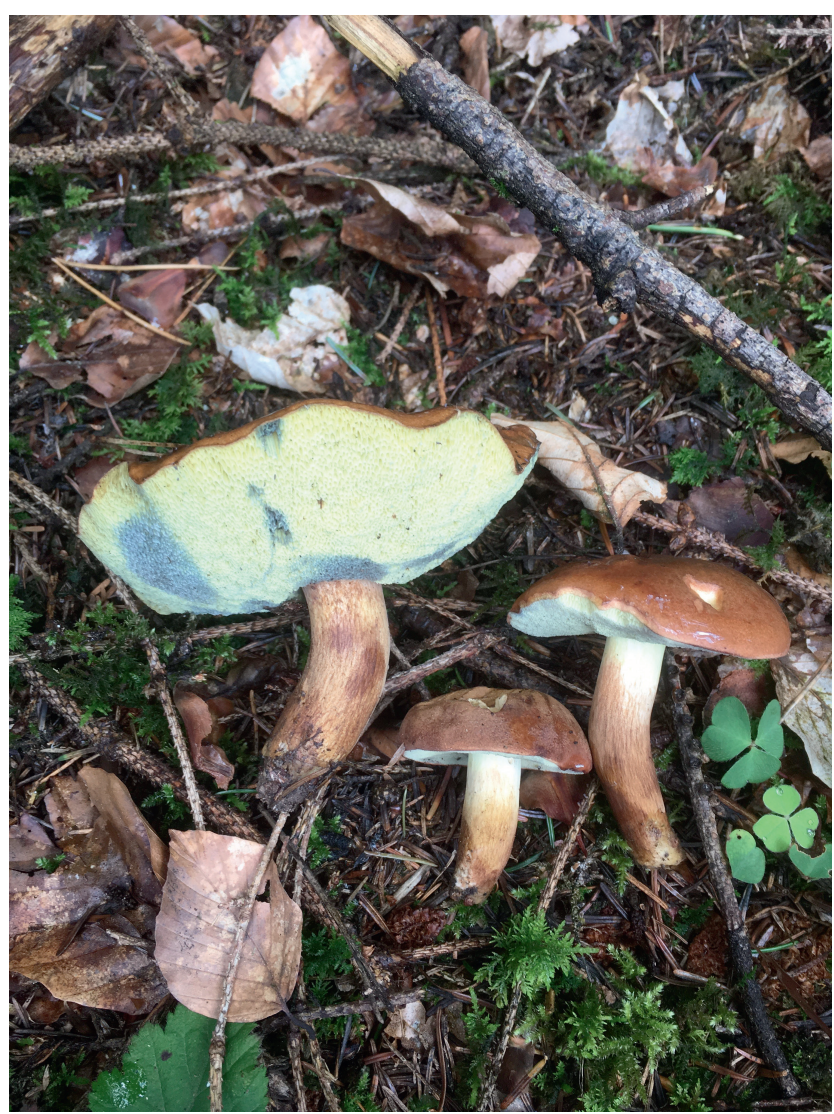

Abbildung 2. Röhren: Imleria badia (Maronenröhrling). Foto: Katharina Schenk-Jäger

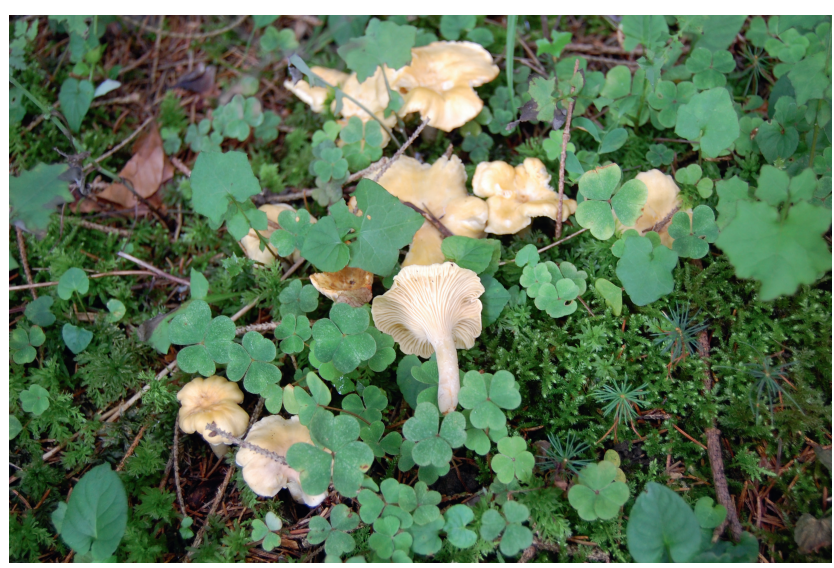

Abbildung 4. Leisten: Cantharellus cibarius (Eierschwamm).

Foto: Katharina Schenk-Jäger

teilung nach der Latenzzeit zwischen Mahlzeit und Symptombeginn [4].

In die Gruppe der Syndrome mit kurzer Latenz (bis 4 Stunden) fallen eher harmlose Vergiftungssyndrome, im Vordergrund steht dabei die Unverträglichkeit. Ebenfalls eine kurze Latenzzeit weist die Fliegenpilzvergiftung auf. Oft handelt es sich hierbei um intentionelle Expositionen, nicht selten in Kombination mit anderen Drogen.

Die Gruppe mit langer Latenzzeit umfasst Syndrome, bei denen Beschwerden nach sechs Stunden bis drei Wochen auftreten. Hier besteht das Risiko irreparabler Organschäden. Im Fall der Amatoxinvergiftung steht das Leberversagen im Vordergrund, bei der Orellaninvergiftung 
das Nierenversagen. In Tabelle 1 sind die wichtigsten und häufigsten Syndrome geordnet von kurzer bis langer Latenzzeit aufgeführt.

\section{Abklärungsstrategie: Anamnese und Labordiagnostik}

Bei Personen mit Beschwerden nach Pilzkonsum ist die Anamnese das wichtigste Instrument, um banale von potenziell lebensgefährlichen Symptomen zu unterscheiden. Bei den potenziell lebensgefährlichen Pilzvergiftungen gilt es vor allem, diejenige mit amatoxinhaltigen Pilzen rasch und zuverlässig zu erkennen, da es nur für diese Vergiftung ein spezifisches Antidot gibt, dessen Wirksamkeit entscheidend davon abhängt, wie zeitnah es verabreicht wird. Es sind anamnestisch wichtige Fragen zu klären (Tabelle 2).

Bevor die Resultate weiterer Abklärungen und Laboruntersuchungen eintreffen, muss bei Verdacht auf eine Vergiftung mit amatoxinhaltigen Pilzen umgehend mit der antidotalen Therapie begonnen werden, da deren Erfolg massgeblich davon abhängt, wie zeitnah sie begonnen wird.

Die weitere Diagnostik richtet sich nach dem klinischen Zustandsbild und der Risikobeurteilung anhand

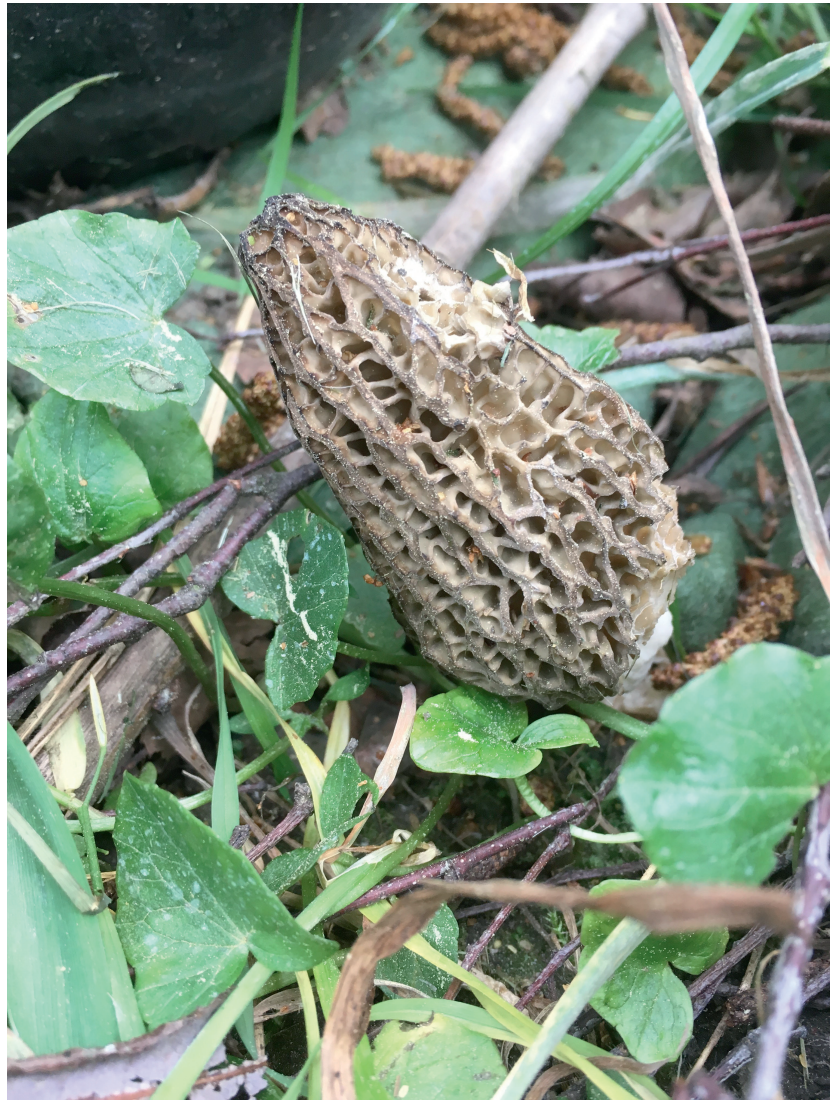

Abbildung 5. Morchelartig: Morchella conica (Spitzmorchel). Foto: Katharina Schenk-Jäger

Tabelle 1. Häufigste und wichtigste Pilzvergiftungssyndrome

\begin{tabular}{|c|c|c|c|c|}
\hline Syndrom - Toxine & Pilze (Fruchtschicht) & Latenz & Leitsymptome & Komplikationen \\
\hline Unverträglichkeit & $\begin{array}{l}\text { Speisepilze (Bsp. Nebelkappe, } \\
\text { Hallimasch) } \\
\text { (R, La, Le, Mo, a) }\end{array}$ & $\begin{array}{l}\text { Meist 1-4 Stunden, } \\
\text { selten länger }\end{array}$ & $\begin{array}{l}\text { Nausea, Völlegefühl, } \\
\text { Erbrechen, Diarrhö }\end{array}$ & $\begin{array}{l}\text { Meist keine, ggf. Dehydrierung } \\
\text { und prärenale, reversible } \\
\text { Nierenfunktionsstörung }\end{array}$ \\
\hline $\begin{array}{l}\text { Gastrointestinales } \\
\text { Frühsyndrom } \\
\text { (diverse Toxine) }\end{array}$ & $\begin{array}{l}\text { Beispiele } \\
\text { Satansröhrling, Tigerritterling, } \\
\text { Grünblättriger Schwefelkopf } \\
(\mathrm{R}, \mathrm{La}, \mathrm{a})\end{array}$ & Meist 1-4 Stunden & $\begin{array}{l}\text { Heftiges Erbrechen } \\
\text { und Diarrhö }\end{array}$ & $\begin{array}{l}\text { Gehäuft Dehydrierung und } \\
\text { darauffolgende Nierenfunk- } \\
\text { tionsstörung }\end{array}$ \\
\hline $\begin{array}{l}\text { Pantherina-Syndrom } \\
\text { (lbotensäure, Muszimol) }\end{array}$ & $\begin{array}{l}\text { Fliegenpilz, Pantherpilz } \\
\text { (ausschliesslich La) }\end{array}$ & 0,5-2 Stunden & $\begin{array}{l}\text { Agitation, Halluzinationen, } \\
\text { Tachykardie, Mundtrockenheit, } \\
\text { selten Koma }\end{array}$ & $\begin{array}{l}\text { Rhabdomyolyse, (Selbst-) } \\
\text { Verletzung }\end{array}$ \\
\hline $\begin{array}{l}\text { Muskarinsyndrom } \\
\text { (Muskarin) }\end{array}$ & $\begin{array}{l}\text { Kleine weisse Trichterlinge, } \\
\text { Risspilze (ausschliesslich La) }\end{array}$ & 0,5-2 Stunden & $\begin{array}{l}\text { Hypersalivation, Lakrimation, } \\
\text { Bradykardie, Diarrhö }\end{array}$ & $\begin{array}{l}\text { Lungenödem im Rahmen } \\
\text { des cholinergen Syndroms }\end{array}$ \\
\hline $\begin{array}{l}\text { Phalloides-Syndrom } \\
\text { (Amatoxine) }\end{array}$ & $\begin{array}{l}\text { Grüner Knollenblätterpilz, } \\
\text { Kegelhütiger Knollenblätterpilz, } \\
\text { Giftschirmlinge, } \\
\text { Gifthäubling } \\
\text { (ausschliesslich La) }\end{array}$ & 6-15 Stunden & $\begin{array}{l}\text { Initial oft heftiges Erbrechen, } \\
\text { Diarrhö. Nach einem oligo- } \\
\text { symptomatischen Intervall } \\
\text { Leberversagen }\end{array}$ & $\begin{array}{l}\text { Zusätzlich Nierenversagen, } \\
\text { Gerinnungsstörung, Leber- } \\
\text { transplantation, letaler } \\
\text { Verlauf möglich }\end{array}$ \\
\hline $\begin{array}{l}\text { Morchellasyndrom } \\
\text { (unbekannt) }\end{array}$ & $\begin{array}{l}\text { Frische Speisemorcheln, } \\
\text { Spitzmorchel } \\
\text { (ausschliesslich Mo, } \\
\text { Frühjahrspilze) }\end{array}$ & 6-12 Stunden & $\begin{array}{l}\text { Zerebelläre Symptome } \\
\text { mit Ataxie, Schwindel, } \\
\text { Doppelbildern }\end{array}$ & Keine bekannt \\
\hline $\begin{array}{l}\text { Orellaninsyndrom } \\
\text { (Orellanin) }\end{array}$ & $\begin{array}{l}\text { Orangefuchsiger Rauhkopf, } \\
\text { Spitzbuckliger Rauhkopf, } \\
\text { (ausschliesslich La) }\end{array}$ & 3 Tage bis 3 Wochen & $\begin{array}{l}\text { Unspezifische Initialsymptome } \\
\text { (Kopfweh, Malaise), gefolgt } \\
\text { von Durst, Oligo- oder Polyurie, } \\
\text { Flankenschmerzen als Ausdruck } \\
\text { des Nierenversagens }\end{array}$ & Irreversibles Nierenversagen \\
\hline
\end{tabular}


Tabelle 2. Anamnese bei Verdacht auf Pilzvergiftung

\begin{tabular}{|c|c|c|}
\hline & Hinweis auf nicht-amatoxinhaltige Pilze & Hinweis auf amatoxinhaltige Pilze \\
\hline $\begin{array}{l}\text { Wurden die Pilze einer Pilzfachperson zur Kontrolle } \\
\text { vorgelegt? }\end{array}$ & Kontrollierte Pilze & Nicht kontrollierte Pilze \\
\hline $\begin{array}{l}\text { Welche Fruchtschicht hatten die Pilze: Röhren, } \\
\text { Lamellen, Leisten, andere? }\end{array}$ & Röhren, Leisten, morchelartig & Lamellen \\
\hline Mit welcher Latenz sind die Symptome aufgetreten? & $\begin{array}{l}\text { Innerhalb von } 4 \text { Stunden } \\
\text { (Cave Mischpilze!) }\end{array}$ & Länger als 6 Stunden \\
\hline Haben alle Tischgenossen dieselben Symptome? & Nur einzelne Personen & Alle haben dieselben Symptome \\
\hline Welcher Art sind die Symptome? & $\begin{array}{l}\text { Leichte Symptome wie Völlegefühl, } \\
\text { Nausea }\end{array}$ & $\begin{array}{l}\text { Heftige gastroenteritische Symptome mit } \\
\text { Brechdurchfällen, später Leberversagen, } \\
\text { welches sich u. a. in Ikterus, Malaise äussert }\end{array}$ \\
\hline
\end{tabular}

der Anamnese. Im Fall von gastroenteritischen Symptomen sicher benigner Ursache reicht meist die Elektrolytkontrolle, bei älteren Personen sollten zusätzlich die Nierenwerte kontrolliert werden, da hier das Risiko einer Nierenfunktionsstörung wegen Dehydrierung besteht. Bei Personen mit neurologischen Symptomen sollten zusätzlich die CK bestimmt werden, um eventuelle Folgen eines Liegetraumas zu erkennen. Sollte sich jedoch aufgrund der Anamnese mit Konsum nicht-kontrollierter Lamellenpilze und Symptombeginn >6 Stunden der Verdacht auf eine Amatoxinvergiftung erhärten, sind folgende Laborwerte zu erheben: neben Elektrolyten auch Leber- und Nierenwerte sowie Gerinnungsstatus. Amatoxine werden im Normalfall im Urin nachgewiesen, neben der herkömmlichen ELISA-Methode setzt sich zunehmend die Analytik mittels LC-MS/MS durch.

Zur weiteren Risikoabschätzung kann neben der Suche nach Amatoxinen im Urin die Expertise von NotfallPilzexpertinnen und -experten beigezogen werden. Diese speziell ausgebildeten Pilzfachleute sind in der Lage, Rüstabfälle und Mahlzeitenresten zu begutachten und einzuschätzen, ob im Gericht amatoxinhaltige Pilze vorhanden sind. Unter Umständen kann auch ein Foto der verzehrten Pilze eine erste Einschätzung ermöglichen.

Da es innerhalb einer Tischgemeinschaft dosisabhängig zu einem gestaffelten Auftreten von Symptomen kommen kann, müssen bei Verdacht auf Amatoxinvergiftung sämtliche Tischgenossen behandelt werden, selbst wenn sie (noch) keine Symptome aufweisen.

\section{Fallbericht 1}

\section{Anamnese der Patientin}

Die 47-jährige Patientin hat am Vortag im Wald zwei bis drei Arten Pilze gesammelt, ohne diese danach an einer Pilzkontrollstelle kontrollieren zu lassen. Sie hat die Pilze dann am selben Tag abends in der Bratpfanne in Butter angebraten, mit Rahm verfeinert und gegessen. Am
Morgen des nächsten Tages zeigte die Patientin Nausea und Erbrechen, wenige Stunden später auch Diarrhö. Es folgte der Anruf bei Tox Info Suisse (Tel. 145, Schweiz) ca. 36 Stunden nach der Mahlzeit. Die Patientin konnte keine Auskunft geben, ob es sich um Lamellen- oder Röhrenpilze handelte, welche sie gesammelt bzw. gegessen hat. Von dem Pilzgericht waren noch Reste übrig, es haben keine anderen Personen davon gegessen.

\section{Weg zur Diagnose/Kommentar}

Da die Pilze nicht kontrolliert worden sind, die Einnahme von Lamellenpilzen nicht ausgeschlossen werden konnte und eine Latenzzeit von rund zwölf Stunden zwischen Mahlzeit bis zum Symptombeginn bestand, konnte eine mögliche Amatoxinvergiftung nicht ausgeschlossen werden.

Tox Info Suisse empfahl zu diesem Zeitpunkt eine Hospitalisation der Patientin zur repetitiven Gabe von Aktivkohle zwecks Unterbrechung des enterohepatischen Kreislaufs, Antidotgabe (Silibinin), Bestimmung des Amanitins im Urin sowie zeitnahen Identifikation der Reste des Pilzgerichts durch eine Notfall-Pilzexpertin der Vereinigung amtlicher Pilzkontrollorgane der Schweiz (www.vapko.ch).

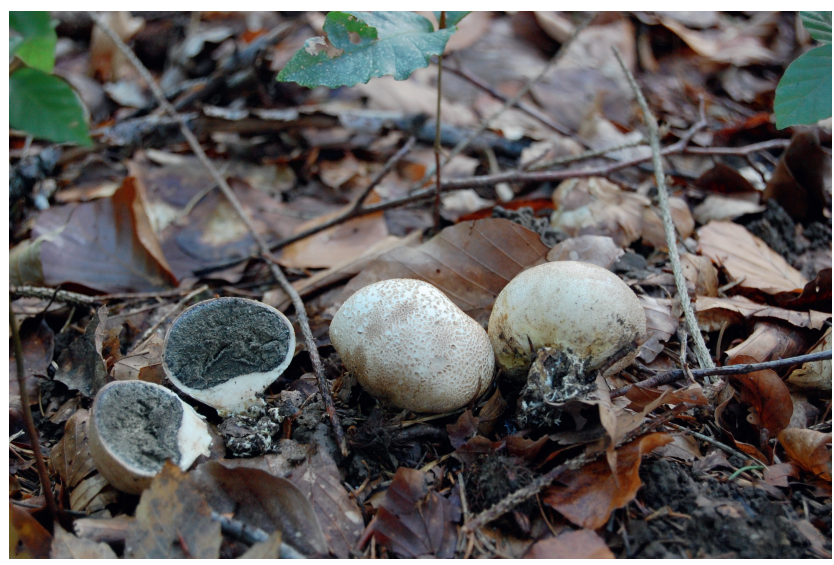

Abbildung 6. Andere: Scleroderma areolatum (Gefelderter Kartoffelbovist). Foto: Katharina Schenk-Jäger 


\section{Befunde der Patientin bei Eintritt ins Krankenhaus}

\section{Status}

47-jährige afebrile Patientin in leicht reduziertem Allgemeinzustand, BMI 32,2 kg/m², GCS 15, BD 143/77 mmHg, Puls 87/min, leicht ikterisch, Abdomen weich mit diffuser Druckdolenz über allen Quadranten, keine Organomegalien, rege Darmgeräusche.

\begin{tabular}{llll}
\multicolumn{2}{l}{ Labor (Maximalwerte) } \\
ASAT & $\mathbf{1 3 4 2 0 ~ U / 1}$ & $\uparrow \uparrow \uparrow$ & $(<35)$ \\
ALAT & $\mathbf{8 9 6 7} \mathrm{U} / 1$ & $\uparrow \uparrow \uparrow$ & $(<35)$ \\
Bilirubin total & $\mathbf{9 1 ~ u m o l / 1}$ & $\uparrow \uparrow$ & $(<21)$ \\
LDH & $\mathbf{1 8 2 9 6 \mathrm { U } / 1}$ & $\uparrow \uparrow \uparrow$ & $(240-280)$ \\
GGT & $\mathbf{7 4} \mathrm{U} / 1$ & $\uparrow$ & $(<40)$ \\
Alk. Phosphatase & $84 \mathrm{U} / 1$ & & $(35-104)$ \\
INR & $\mathbf{2 , 1}$ & $\uparrow$ & $(<1,2)$ \\
Thrombinzeit & $\mathbf{2 4} \mathrm{sek}$. & $\uparrow$ & $(<22)$ \\
aPTT & $28 \mathrm{sek}$. & & $(24-36)$ \\
Faktor V & $\mathbf{1 0} \%$ & $\downarrow \downarrow$ & $(50-150)$ \\
Faktor XIII & $132 \%$ & & $(70-140)$ \\
Ammoniak & $\mathbf{6 5} \mathrm{umol} / 1$ & $\uparrow$ & $(9-30)$ \\
Kreatinin & $55 \mathrm{umol} / 1$ & & $(44-80)$ \\
Harnstoff & $4,7 \mathrm{mmol} / 1$ & & $(2,14-7,14)$ \\
Natrium & $139 \mathrm{mmol} / 1$ & & $(136-145)$ \\
Kalium & $3,6 \mathrm{mmol} / 1$ & & $(3,3-4,5)$
\end{tabular}

Erweitertes Labor am Folgetag

Alpha-Amanitin

(Urin) 9,0 ug/1 (negativ)

\section{Weitere Befunde}

Notfall-Pilzexpertin (VAPKO): Identifikation von Amanita phalloides (Grüner Knollenblätterpilz) in den Resten des Pilzgerichts.

\section{Therapie}

Repetitive Gabe von Aktivkohle (Carbovit ${ }^{\circledR}$ ) oral. Antidotale Therapie mit Silibinin i.v. $\left(\right.$ Legalon $\left.^{\circledR}\right)$. N-Acetylcystein i.v. (Fluimucil $^{\circledR}$ ) als Hepatoprotektivum analog Schema Prescott bei Paracetamolintoxikation.

\section{Weiterer Verlauf}

Verlegung von Regionalspital in Zentrumsspital an Tag 3 nach der Mahlzeit bei massiv progredienten Leberwerten. Im weiteren Verlauf Anstieg der Transaminasen mit einem Maximalwert nach 76 h sowie einem Nadir des Faktor V nach 64 h (s. Labor). Keine klinischen Hinweise für eine Encephalopathie, sodass die Kriterien für eine SuperUrgent-Listung zur Lebertransplantation nicht erfüllt wurden. Alpha-Amanitin an Tag 4 nicht mehr nachweisbar, daraufhin Sistierung der Gabe von Aktivkohle und Silibi- nin. Gabe von N-Acetylcystein bis zur Normalisierung der Leberwerte. Vollständige Erholung.

\section{Fallbericht 2}

\section{Anamnese des Patienten}

Der 31-jährige Patient hat morgens bei einem Waldspaziergang direkt nebeneinander zwei Hexenröhrlinge gefunden. Er hat diese mitgenommen und ein Foto davon einem Freund geschickt, welcher sich mit Pilzen «etwas» auskenne. Dieser habe bestätigt, dass es sich um einen Hexenröhrling handle. Der Patient hat einen der beiden Pilze daraufhin gerüstet, in Scheiben geschnitten, angebraten und zum Mittagessen verspeist. Etwa zwei Stunden später bekam er starke Bauchschmerzen und Nausea und musste mehrfach erbrechen. Eine weitere Stunde später zeigte er zusätzlich wiederholt wässrige Diarrhö. Bei persistierenden gastrointestinalen Symptomen suchte er abends die Notfallstation des Krankenhauses auf. Der behandelnde Arzt kontaktierte daraufhin Tox Info Suisse (Notfallnummer Tel. 145. Schweiz). Der Patient konnte mit Sicherheit sagen, dass es sich bei den zwei gesammelten Pilzen um Röhrenpilze und nicht um Lamellenpilze handelte.

\section{Weg zur Diagnose/Kommentar}

Das gefährliche hepatotoxische Amatoxin kommt in Röhrenpilzen nicht vor. Röhrenpilze können essbar oder ungeniessbar bzw. giftig sein. Bei gastrointestinalen Symptomen nach Konsum von Röhrenpilzen gibt es zwei mögliche Erklärungen:

1. Bei ungeniessbaren/giftigen Röhrenpilzen:

\section{Gastrointestinales Frühsyndrom}

Latenzzeit: Innerhalb maximal $4 \mathrm{~h}$.

Symptome: Abdominalschmerzen, Übelkeit, Erbrechen, Durchfall.

Therapie: symptomatisch.

2. Bei falsch zubereiteten oder bakteriell kontaminierten essbaren Röhrenpilzen:

\section{Indigestionssyndrom}

Latenzzeit: Meist innerhalb von $4 \mathrm{~h}$, gelegentlich aber erst nach 8-12 h.

Symptome: Abdominalschmerzen, Übelkeit, Erbrechen, Durchfall.

Therapie: symptomatisch.

Bei Einnahme von Röhrenpilzen bringt die Identifikation des Pilzes durch eine Notfall-Pilzexpertin/einen Notfall-Pilzexperten definitive Sicherheit bezüglich der eingenommenen Pilzart, ist jedoch nicht zwingend notwendig. Die Therapie bleibt in jedem Fall symptomatisch. 


\section{Befunde des Patienten bei Eintritt ins Krankenhaus}

\section{Status}

31-jähriger afebriler Patient in leicht reduziertem Allgemeinzustand. GCS 15, BD 105/72 mmHg, Puls 109/min. Abdomen weich, rege Darmgeräusche über allen 4 Quadranten, kein Peritonismus.

\begin{tabular}{llll}
\multicolumn{2}{l}{ Labor (Maximalwerte) } \\
Natrium & $140 \mathrm{mmol} / 1$ & & $(136-145)$ \\
Kalium & $3,1 \mathrm{mmol} / 1$ & $\downarrow$ & $(3,3-4,5)$ \\
Kreatinin & $75 \mathrm{umol} / 1$ & & $(44-80)$ \\
Hämoglobin & $130 \mathrm{~g} / \mathrm{l}$ & & $(140-180 \mathrm{~g} / \mathrm{l})$ \\
Leukocyten & $10,1 \mathrm{G} / 1$ & $(3,5-10,5)$ \\
CRP & $3 \mathrm{mg} / 1$ & $(<5 \mathrm{mg} / \mathrm{l})$ \\
ASAT & $32 \mathrm{U} / 1$ & $(<35)$ \\
ALAT & $40 \mathrm{U} / 1$ & $(<35)$
\end{tabular}

\section{Weitere Befunde}

Der mitgebrachte nicht verspeiste Pilz konnte mithilfe eines beigezogenen Notfall-Pilzexperten als Satansröhrling (Rubroboletus satanas) identifiziert werden.

\section{Therapie}

Symptomatische Therapie mit Antiemetika, Flüssigkeitsund Elektrolytkorrektur.

Eine spezifische bzw. antidotale Therapie ist nicht nötig, wenn definitiv keine Lamellenpilze involviert waren.

\section{Weiterer Verlauf}

Unter symptomatischer Therapie rasche und vollständige Erholung des Patienten innerhalb weniger Stunden.

\section{Kommentar}

In diesem Fall handelte es sich um eine Verwechslung des Hexenröhrlings mit dem Satansröhrling.

Der bei korrekter Zubereitung geniessbare Hexenröhrling wird von Laien gerne mit dem giftigen Satansröhrling verwechselt, welcher ein gastrointestinales Frühsyndrom verursacht.

Wenn es sich bei den eingenommenen Pilzen ausschliesslich um Röhrenpilze handelt, ist eine weitere mykologische Abklärung nicht zwingend notwendig, da diese keine therapeutischen Konsequenzen hat.

ACHTUNG: In einem unkontrollierten Mischgericht (Röhrenpilze und Lamellenpilze) können ungeniessbare Pilze, verdorbene Speisepilze und amanitinhaltige Pilze gemeinsam vorkommen. Die kurze Latenzzeit bis zum Auftreten von Symptomen kann die typische Symptomatik der Amatoxinvergiftung maskieren. Deshalb bei Mischgerichten immer Amatoxin ausschliessen!

\section{Diagnostik}

\section{Klinischer Untersuch}

\section{Labor}

Bei Verdacht auf eine Amatoxinvergiftung soll neben der Bestimmung der Leber- und Nierenwerte und Gerinnungsparameter auch die Analytik auf Amatoxine im Urin veranlasst werden. Am aussagekräftigsten ist die Bestimmung im Urin 6-60 Stunden nach der Mahlzeit. Es stehen zwei Verfahren zur Verfügung: herkömmlich mittels ELISA und seit kurzem auch mittels Lc-MS/MS. Beide Verfahren sind zeitaufwändig und stehen nicht in jedem Labor rund um die Uhr zur Verfügung.

Notfall-Pilzexpertinnen und -experten können in Rüstabfällen und Mahlzeitenresten makroskopisch Pilzfragmente bestimmen. Gelegentlich kommt auch das Mikroskop zum Zug oder es werden chemische Farbreaktionen herangezogen.

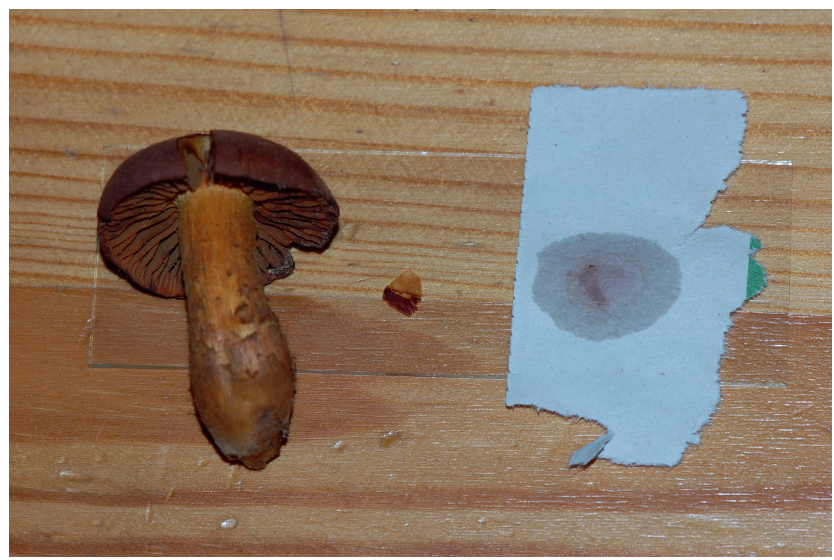

Abbildung 7. Makroskopische Reaktion: Orellanintest nach Pöder/Moser: Eisenchloridlösung zur Anfärbung des Orellanins [5]. Foto: Katharina Schenk-Jäger

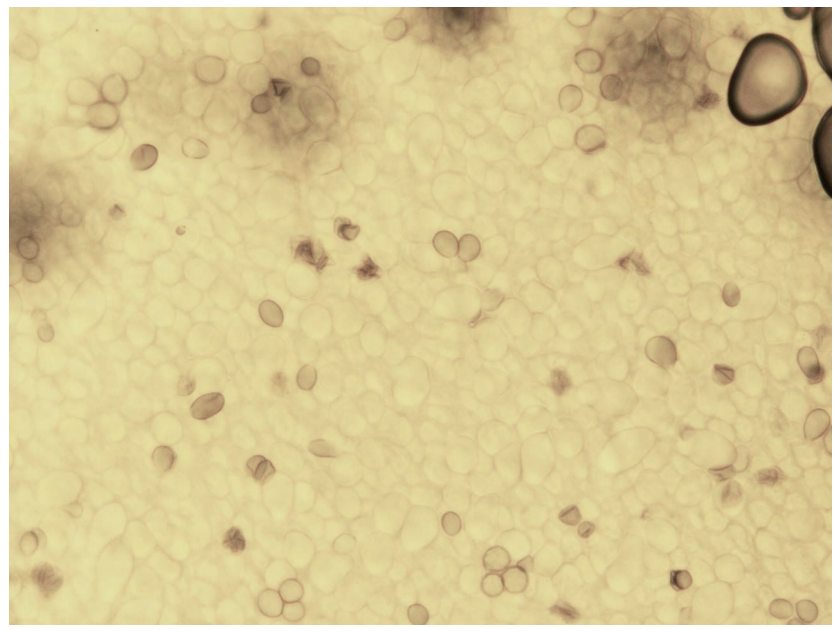

Abbildung 8. Sporen von Amanita phalloides angefärbt mit Melzers Reagens (= Amyloidreaktion): lodlösung in Chloralhydrat, wobei das lod die Stärke blau-violett anfärbt [5].

Foto: Michel Schneider 
Tabelle 3. Pilzarten, ihr Speisewert und mögliche Doppelgänger

\begin{tabular}{|c|c|c|c|}
\hline Lateinischer Name & Volkstümlicher Name & Speisewert & Kann verwechselt werden mit (Auswahl) \\
\hline Amanita phalloides & Grüner Knollenblätterpilz & Giftig & Champignons, Täublingen \\
\hline Amanita pantherina & Pantherpilz & Giftig & Perlpilz, kleinen Steinpilzen \\
\hline Amanita muscaria & Fliegenpilz & Giftig & Kaiserling \\
\hline Boletus edulis & Steinpilz & Speisepilz & Kleinen Pantherpilzen \\
\hline Clitocybe nebularis & Nebelkappe & Speisepilz (bedingt) & Riesenrötling \\
\hline Entoloma sinuatum & Riesenrötling & Giftig & Nebelkappe \\
\hline Hypholoma fasciculare & Grünblättriger Schwefelkopf & Giftig & Stockschwämmchen \\
\hline Armillaria mellea & Hallimasch & Speisepilz (bedingt) & Nadelholz-Gifthäubling \\
\hline Kuehneromyces mutabilis & Stockschwämmchen & Speisepilz & $\begin{array}{l}\text { Nadelholz-Gifthäubling, Grünblättrigem } \\
\text { Schwefelkopf }\end{array}$ \\
\hline Galerina marginata & Nadelholz-Gifthäubling & Giftig & Stockschwämmchen \\
\hline Tricholoma pardinum & Tigerritterling & Giftig & Erdritterling \\
\hline Tricholoma terreum & Erdritterlingq & Speisepilz & Tigerritterling \\
\hline Rubroboletus satanas & Satansröhrling & Giftig & Hexenröhrling \\
\hline Suillellus luridus & Netzstieliger Hexenröhrling & Speisepilz & Satansröhrling \\
\hline Neoboletus erythropus & Flockenstieliger Hexenröhrling & Speisepilz & Satansröhrling \\
\hline Cortinarius rubellus & Spitzbuckliger Rauhkopf & Giftig & Kahlköpfen \\
\hline Cortinarius orellanus & Orangefuchsiger Rauhkopf & Giftig & Sehr kleinen Eierschwämmen \\
\hline Cantharellus cibarius & Eierschwamm & Speisepilz & Sehr kleinen Rauhköpfen \\
\hline Lepiota sp. & Giftschirmlinge & Giftig & Parasol \\
\hline Macrolepiota procera & Parasol & Speisepilz & Giftschirmlingen \\
\hline Inocybe sp. & Risspilze & Giftig & Mehlräsling \\
\hline Clitopilus prunulus & Mehlräsling & Speisepilz & Risspilzen \\
\hline
\end{tabular}

Abbildungen 7 und 8 zeigen chemische Farbreaktionen, welche von Mykologen gerne verwendet werden.

\section{Weitere mykologische Gesichtspunkte}

Die Liste möglicher Verwechslungen zwischen Speise- und Giftpilzen ist lang. Wie grob die Fehler sind, hängt vom Vorwissen des Sammlers ab. In der folgenden Liste (Tab. 3) sind einige Pilze aufgeführt, die aufgrund habitueller Ähnlichkeiten verwechselt werden können. Speziell heikel ist der Verzehr kleiner Fruchtkörper, bei welchen noch nicht alle Merkmale gut sichtbar ausgebildet sind. So können zum Beispiel kleine Steinpilze mit kleinen Pantherpilzen verwechselt werden, wenn noch keine freie Sicht auf die sich bildenden Röhren, resp. Lamellen möglich ist. Im Verbreitungsatlas der Schweizer Pilze, welcher von der Eidgenössischen Forschungsanstalt für Wald, Schnee und Landschaft (WSL) herausgegeben wird, können die Verbreitungsgebiete einzelner Pilzarten abgefragt werden [6].

\section{Therapie und Prognose, Prävention}

\section{Therapie}

Die Therapie einer Intoxikation umfasst drei Pfeiler: Dekontamination zur Verhinderung der Aufnahme resp. Beschleunigung der Elimination der Toxine, antidotale Therapie und supportive Massnahmen. Das gilt auch für Pilzvergiftungen.

1. Dekontamination: Pilzgifte binden an Aktivkohle. Daher kann bei erhaltener Vigilanz die Gabe von Aktivkohle $(1 \mathrm{~g} / \mathrm{kg} \mathrm{KG})$ erwogen werden. Bei Verdacht auf Amatoxinvergiftung kann die Aktivkohle zudem wiederholt verabreicht werden zur Unterbrechung des enterohepatischen Kreislaufes.

2. Ein spezifisches Antidot gibt es im Fall einer Pilzvergiftung nur für die Amatoxine. Silibinin, der Wirkstoff aus der Mariendistel (Silybum marianum), verhindert die Aufnahme der Amatoxine in die Hepatozyten und kann daher die Hepatotoxizität reduzieren oder gar verhindern. Auch wenn der Nutzen zum Teil kontrovers diskutiert wird, erscheinen die Hinweise auf eine Wirksamkeit 
Tabelle 4. Therapieoptionen der häufigsten und wichtigsten Pilzvergiftungssyndrome

\begin{tabular}{llll}
\hline Syndrom - Toxine & Toxine - Wirkmechanismus & Therapie & Analytik, Laborkontrollen \\
\hline Unverträglichkeit & $\begin{array}{l}\text { Lokale Reizerscheinung wegen ungenügend } \\
\text { gekochter Speisepilze oder zu grosser Menge }\end{array}$ & $\begin{array}{l}\text { Rehydrierung, Antiemetika, } \\
\text { Antidiarrhoika }\end{array}$ & -
\end{tabular}

Gastrointestinales

Frühsyndrom

Pantherinasyndrom

Muskarinsyndrom

Lokale Reizung der Mukosa des Gastrointestinaltraktes

Ibotensäure und Muszimol sind den Neurotransmittern Glutamat und GABA strukturell ähnlich. Je nach anteilmässigem Verhältnis kann die stimulierende (Glutamat) oder die sedierende Wirkung (GABA) überwiegen

Muskarin vermittelt an der Synapse anstelle des Acetylcholins die Reizübertragung. Muskarin wird durch die Acetylcholinesterase nicht abgebaut und kann deshalb eine parasympathische Dauererregung hervorrufen. Passiert die Blut-Hirn-Schranke nicht

Phalloides-Syndrom

Amatoxine sind bicyclische Oktapeptide. Hauptwirkung ist die Blockierung der RNAPolymerase II. Dadurch wird die Transkription der mRNA unterbrochen, wodurch die Biosynthese vieler Proteine blockiert wird. Histologisches Bild einer zentrilobulären Nekrose

Morchellasyndrom Unbekannte(s) Toxin(e) mit zerebellären
Symptomen. Scheint nur in frisch
zubereiteten Morcheln vorzukommen, nicht
aber in getrockneten Morcheln

Orellaninsyndrom

Mechanismus nicht vollständig geklärt. Orellanin führt zu oxidativem Stress wegen Bildung reaktiver Sauerstoffradikale, worauf die Proteinbiosynthese blockiert wird. Histologisches Bild einer schweren interstitiellen Fibrose, interstitiellem Ödem und tubulärer Nekrose

Rehydrierung, Antiemetika, Antidiarrhoika, ggf. Elektrolytkorrektur

Sedation mit Benzodiazepinen

Elektrolytkontrolle

Elektrolytkontrolle, CK

Rehydrierung, Atropin

Elektrolytkontrolle

\section{- Spezifisches Antidot: Silibinin (Legalon SIL $®)$ i.v. - 5 mg/kg KG 6-stündlich (Tagesdosis $20 \mathrm{mg} / \mathrm{kg} \mathrm{KG}$ ) in Glucose $5 \%$ oder $\mathrm{NaCl}$ 0,9\% über jeweils $2 \mathrm{~h}$. CAVE: Beginn bereits bei Verdacht und vor Resultaten der Analytik! \\ - Supportive Therapie: N-Acetyl- cystein nach Prescott als hepatoprotektive Massnahme, konsequente Rehydrierung}

Symptomatische Therapie, falls nötig

Elektrolytkorrektur, Azidosekorrektur, Flüssigkeitsbilanzierung, Hämodialyse, im Verlauf ggf. Nierentransplantation
Amatoxine im Urin nachweisbar 6-60 Stunden nach Mahlzeit Laborkontrollen: Elektrolyte, Leber- und Nierenwerte, Gerinnungsstatus
Orellanin-Nachweis im Nierenpunktat bis 6 Monate nach Exposition möglich (Uni Innsbruck), Elektrolytkontrolle, Nierenwerte stark und die Gefahr von unerwünschten Arzneimittelwirkungen ist gering, sodass Tox Info Suisse diese Therapie empfiehlt [7].

Supportive Massnahmen umfassen die enterale oder parenterale Rehydrierung, antiemetische Therapie, Elektrolytkorrektur und im Fall einer Muskarinvergiftung die Verabreichung von Atropin. Als hepatoprotektive Massnahme kommt N-Acetylcystein infrage [8]. Ein durch Orellanin verursachtes Nierenversagen bedingt oft den Einsatz eines Nierenersatzverfahrens, nicht selten bis zur erfolgreichen Organtransplantation. In Tabelle 4 sind nach Syndrom die jeweiligen spezifischen und supportiven Massnahmen zusammen mit Wirkmechanismus und Analytik aufgeführt.

Es kann vorkommen, dass in der erstversorgenden Praxis oder Notfallstation nicht alle Möglichkeiten zur Behandlung einer möglichen (Amatoxin-)Vergiftung vorhanden sind. Dann ist es wichtig, dass diejenigen Massnahmen, die zur Verfügung stehen, eingeleitet werden und die Patientin/der Patient danach verlegt wird. Nur schon eine erste Dosis Aktivkohle kann wertvoll sein, um Pilzgifte rasch zu binden.

\section{Prognose}

\section{Prognostische Kriterien}

Während die Prognose bei den Pilzvergiftungen mit kurzer Latenz in aller Regel gut ist, kann eine Vergiftung mit amatoxin- oder orellaninhaltigen Pilzen zu einem irreversiblen Leber- resp. Nierenversagen führen.

Die Prognose einer Amatoxinvergiftung lässt sich anhand folgender Parameter ableiten: Die Prognose ist schlecht bei einer Latenz von sechs Stunden zwischen Mahlzeit und Beginn der Symptome, bei einem späten Beginn der antidotalen Therapie und Dekontamination, sowie bei Kindern. 


\section{Nützliche Adressen im Notfall}

- Tox Info Suisse: Notrufnummer 145, rund um die Uhr Risikobeurteilung und Empfehlungen zu Abklärungen und Therapie.

- Vapko:Vereinigung Amtlicher Pilzkontrollorgane: www.vapko.ch. Liste der Notfall-Pilzexpertinnen und -experten, die vor Ort Pilzreste (übriggebliebene Exemplare, Rüstabfälle und Mahlzeitenreste) begutachten können.

\section{Weiterführende Auskunftsstellen}

- Toxikologische Informationen:

- Giftinformationszentrum München: Pilzinformationsdienst: https://toxinfo.org/frameset-php_class _3_hauptframe_/pilz/

- Mykologische Informationen:

- VSVP: Verband Schweizerischer Vereine für Pilzkunde: www.vsvp.com. Die Verbandstoxikologin ist unter tox@vsvp.com erreichbar

- Eidgenössische Forschungsanstalt für Wald, Schnee und Landschaft (WSL): Verbreitungsatlas der Pilze in der Schweiz: www.swissfungi.ch

Als Ultima Ratio bleibt in Fällen mit schwerem Verlauf nur die Organtransplantation, wobei sich die Zeit bis zur Transplantation beim Leberversagen durch Amatoxine nur in einem zeitlich sehr limitierten Umfang überbrücken lässt. Die Kriterien zur Indikation der Lebertransplantation bei Amatoxinvergiftung werden kontrovers diskutiert [911]. Die Autoren um Escudié haben als Hauptkriterien die zeitliche Latenz zwischen Exposition und Beginn der gastrointestinalen Symptome, sowie eine Prothrombinzeit von $<10 \%$ am Tag 4 oder später identifiziert. In der Untersuchung von Ganzert wurde die Prothombinzeit von $<10 \%$ in Kombination mit einem erhöhten Kreatinin von $>106 \mu \mathrm{mol} / \mathrm{l}$ an Tag 3-10 als zuverlässigste Messgrösse ermittelt. Andere, für die Entscheidung zur Lebertransplantation entwickelte Modelle wie die King's College oder Clichy's Criteria haben sich als ungeeignet für das Leberversagen im Rahmen einer Amatoxinvergiftung erwiesen.

\section{Prävention}

Die Schweiz verfügt über ein dichtes Netz an Pilzkontrollstellen. Diese sind über die Webseite der Vereinigung Amtlicher Pilzkontrollorgane Vapko (www.vapko.ch) abrufbar. Diese Fachleute stehen zur Verfügung, um Sammelgut vor dem Verzehr zu kontrollieren, sodass der Sammler nur Speisepilze zubereitet. Da selbst passionier- ten Pilzkennern Fehler unterlaufen können, ist die Pilzkontrolle sämtlichen Sammlern dringend empfohlen. Die Pilzkontrolle hat sich in den vergangenen Jahren als einzig zuverlässige Massnahme zur Verhinderung von Pilzvergiftungen erwiesen, da bei Tox Info Suisse keine einzige Vergiftung mit Giftpilzen in kontrolliertem Sammelgut registriert wurde.

\section{Bibliografie}

1. Schenk-Jaeger KM, Rauber-Lüthy C, Bodmer M, Kupferschmidt H, Kullak-Ublick GA, Ceschi A. Mushroom poisoning: A study on circumstances of exposure and patterns of toxicity. Eur J Intern Med. 2012;23:e85-91.

2. Gawlikowski T, Romek M, Satora L. Edible mushroom-related poisoning: A study on circumstances of mushroom collection, transport, and storage. Hum Exp Toxicol. 2015;34(7):718-724.

3. Saviuc P, Harry P, Pulce C, Garnier R, Cochet A. Can morels (Morchella sp.) induce a toxic neurological syndrome? Clin Toxicol (Phila). 2010;48(4):365-372.

4. White J, Weinstein SA, De Haro L, et al. Mushroom poisoning: A proposed new clinical classification. Toxicon. 2019;157:53-65

5. Flammer R. Giftpilze. Aarau/München; AT Verlag: 2014.

6. Eidg. Forschungsanstalt für Wald, Schnee und Landschaft WSL: SwissFungi. Verbreitungsatlas. https://swissfungi.wsl. ch/de/verbreitungsdaten/verbreitungsatlas.html; letzter Zugriff: 10.05.2021

7. Magdalan J, Ostrowska A, Piotrowska A, et al. Benzylpenicillin, acetylcysteine and silibinin as antidotes in human hepatocytes intoxicated with alpha-amanitin. Exp Toxicol Pathol. 2010;62(4):367-73.

8. Liu J, Chen Y, Gao Y, Walline JH, Lu X, Yu S, Zhao L, Ge Z, Li Y. Nacetylcysteine as a treatment for amatoxin poisoning: a systematic review. Clin Toxicol. 2020;58(11):1015-1022.

9. Escudié L, Francoz C, Vinel JP, et al. Amanita phalloides poisoning: Reassessment of prognostic factors and indication for liver transplantation. J Hepatol. 2007;46:466-473.

10. Ganzert M, Felgenhauer N, Zilker T. Indication of liver transplantation following amatoxin intoxication. J Hepatol. 2005; 42:202-209.

11. Enjalbert F, Rapior S, Nouguier-Soulé J, Guillon S, Amouroux N, Cabot C. Treatment of amatoxin poisoning: 20-year retrospective analysis. J Toxicol Clin Toxicol. 2002;40:715-757.

\section{Historie}

Manuskript eingereicht: 05.05.2021

\section{Interessenskonflikte}

Es bestehen keine Interessenskonflikte.

\section{Hinweis}

Katharina Schenk-Jäger ist diplomierte Pilzkontrolleurin und Notfallpilzexpertin Vapko, sowie Vorstandsmitglied des Verbandes Schweizerischer Vereine für Pilzkunde VSVP.

\section{PD Dr. med. Stefan Weiler}

Tox Info Suisse

Freiestrasse 16

8032 Zürich

Stefan.weiler@toxinfo.ch 


\section{CME-Fragen}

\section{CME: Achtung Lamelle! Pilzvergiftungen in der Praxis}

Frage 1: Welche Befunde sprechen eher für eine harmlose Pilzvergiftung (Mehrfachauswahl)

a) Latenzzeit von acht Stunden.

b) Ein Tischgenosse entwickelt nach 30 Minuten Magendarmsymptome, die anderen bleiben beschwerdefrei.

c) Beschwerden treten nach Konsum selbstgesammelter, kontrollierter Pilze auf.

d) 24 Stunden nach Beginn ausgeprägter Brechdurchfälle geht es dem Patienten zwar besser. Bei genauem Hinschauen fällt aber ein Sklerenikterus auf.

e) Eine Woche nach Genuss selbst gesammelter, nicht kontrollierter Pilze treten Flankenschmerzen und Oligourie auf.

Frage 2: Ein Patient stellt sich 18 Stunden nach einer Pilzmahlzeit bei Ihnen im Krankenhaus vor mit rezidivierenden Brechdurchfällen, die zehn Stunden nach Konsum selbstgesammelter Pilze begonnen haben. Wie gehen Sie vor? (Einfachauswahl)

a) Ich kontrolliere Leber- und Nierenwerte. Falls sie normal sind, entlasse ich den Patienten mit symptomatischer Therapie.

b) Sollte es sich bei der Mahlzeit um ein Gericht aus nichtkontrollierten Lamellenpilzen oder Mischpilzen handeln, beginne ich sofort mit Dekontamination und antidotaler Therapie wegen Verdacht auf Amatoxinvergiftung. Sollte das meine Möglichkeiten übersteigen, beginne ich mit denjenigen Massnahmen, die mir zur Verfügung stehen und verlege den Patienten. Ich erkundige mich nach allfälligen Tischgenossen, welche vielleicht noch keine Beschwerden haben, damit auch sie einer Behandlung zugeführt werden können.

c) Ich verlege den Patienten ohne weitere Massnahmen in ein Zentrumsspital, um keine Zeit zu verlieren.

d) Es liegt keine Pilzvergiftung vor, da die Latenzzeit zu lang ist. Ich suche nach einer anderen Ursache der Beschwerden.

e) Da der Patient auf Nachfrage zuverlässig angibt, dass er ausschliesslich Lamellenpilze gegessen hat, belasse ich es bei einer symptomatischen Therapie.

Frage 3: Ein 65-jähriger Mann genehmigt sich an einem Abend in einem Restaurant eine üppige Vorspeise aus frischen Morcheln. Dazu trinkt er ein Glas Weisswein. Später am Abend kommen noch zwei Gläser Rotwein dazu, was seiner Gewohnheit entspricht. Am Folgetag früh morgens wacht der Mann auf, alles dreht sich, er sieht doppelt. Der Gang zur Toilette scheitert an Gangstörungen. Welche Aussage ist korrekt? (Einfachauswahl) a) Der Konsum von Pilzen in einem Restaurant führt nie zu Symptomen, sodass jeglicher Zusammenhang mit dem Pilzgericht abgelehnt werden muss.

b) Selbst wenn der Konsum von Pilzen in einem Restaurant sicher sein sollte, kann es doch zu Verwechslungen mit Giftpilzen kommen. Hier besteht die Gefahr einer Vergiftung durch Giftpilze.

c) Hier handelt es sich um ein Morchellasyndrom, welches nach Konsum grösserer Mengen frisch zubereiteter Morcheln auftreten kann. Dank guter Prognose sind bis auf eine symptomatische Therapie keine weiteren Massnahmen nötig.

d) Es handelt sich um Beschwerden im Rahmen des Alkoholkonsums.

e) Es handelt sich um eine Pilzallergie.

Frage 4: Eine junge Frau sammelt selbst Pilze im Wald. Sie sind gelb-orange und schauen schmackhaft aus. Sie bereitet sie zu und isst sie. Einige Tage später entwickelt sie Übelkeit, Erbrechen, Durchfall. Laborchemisch zeigt die Patientin nur eine leicht erhöhte Alanin-Aminontransferase (ALT), aber eine starke Erhöhung des Serumkreatinins von $7,9 \mathrm{mg} / \mathrm{dl}$ bzw. $689 \mu \mathrm{mol} / 1$ zusammen mit einem plötzlichen Beginn einer Oligurie $(100 \mathrm{ml} / \mathbf{2 4} \mathrm{h})$.

Was ist das? (Einfachauswahl)

a) Orellanus-Syndrom

b) Ein protrahierter Verlauf einer Amatoxinvergiftung

c) Eine Indigestion

d) Keine Pilzvergiftung, da die Latenz zu lange ist

e) Folgen einer Rhabdomyolyse nach Fliegenpilzkonsum

Frage 5: Bei welchen der genannten Gattungen gibt es amatoxinhaltige Pilzarten? (Mehrfachauswahl)
a) Schleierlinge (Cortinarius sp.)
b) Wulstlinge (Amanita sp.)
c) Risspilze (Inocybe sp.)
d) Gifthäublinge (Galerina sp.)
e) Giftschirmlinge (Lepiota sp.)

\section{PD Dr. med. Stefan Weiler}

Pharmakoepidemiologie, Institut für Pharmazeutische Wissenschaften, Departement Chemie und Angewandte

Biowissenschaften

ETH Zürich

Vladimir-Prelog-Weg 1-5/10

8093 Zürich

stefan.weiler@pharma.ethz.ch 


\section{CME-Antworten}

Sie können die Fragen bei unserem Online-Fortbildungsangebot CME unter www.praxis.ch bis spätestens 29. August 2021 lösen. Nach korrekter Beantwortung der Fragen erhalten Sie automatisch eine Bestätigung. Sie können aber auch untenstehenden Antworttalon verwenden. Auflösung und Kommentare erscheinen in der «Praxis» Nr. 11 vom 1. September 2021.

\section{Antworttalon}

Um Ihre Bestätigung für das Bearbeiten der CME zu erhalten, schicken Sie uns bitte den ausgefüllten Antworttalon bis spätestens 29. August 2021 mit einem frankierten und an Sie adressierten Rückumschlag an Hogrefe AG, Redaktion Praxis, Länggass-Strasse 76 , 3012 Bern.

Ihre Antworten zum Thema «CME: Achtung Lamelle! Pilzvergiftungen in der Praxis»

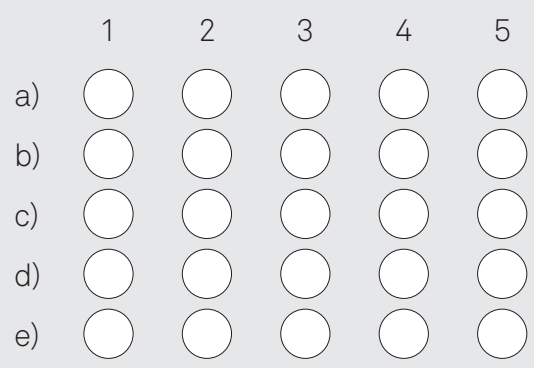

\section{Absender}

Titel/FMH

Name

Vorname

Adresse

PLZ, Ort

Datum

Unterschrift

Ich versichere, alle Fragen ohne fremde Hilfe beantwortet zu haben.

Bei erfolgreich abgeschlossenem Fortbildungsmodul (mind. 60\% der Antworten richtig) kann gemäss Akkreditierungsentscheid der SGAIM 1 Kernfortbildungscredit AIM erworben werden. Es gelten hier die Limitationen für e-Learnings/Zeitschriften: Maximal 8 Kernfortbildungscredits pro Jahr bzw. 24 Kernfortbildungscredits pro 3-Jahres-Periode. Darüber hinausgehende Credits können unbeschränkt als erweiterte Fortbildung angerechnet werden. 